\title{
Rectangular Drawings of Planar Graphs ${ }^{\star}$ (Extended Abstract)
}

\author{
Md. Saidur Rahman ${ }^{1}$, Takao Nishizeki ${ }^{2}$, and Shubhashis Ghosh ${ }^{3}$ \\ 1 Graduate School of Information Sciences, Tohoku University, Aoba-yama 05, \\ Sendai 980-8579, Japan. On leave from Bangladesh University of Engineering and \\ Technology (BUET). saidur@nishizeki.ecei.tohoku.ac.jp \\ 2 Graduate School of Information Sciences, Tohoku University, Aoba-yama 05, \\ Sendai 980-8579, Japan. nishi@ecei.tohoku.ac.jp \\ 3 Department of Computing Science, University of Alberta, Edmonton, Alberta T6G \\ 2E8, Canada. On leave from Bangladesh University of Engineering and Technology \\ (BUET). shubhash@cs.ualberta.ca
}

\begin{abstract}
A plane graph is a planar graph with a fixed embedding. In a rectangular drawing of a plane graph, each vertex is drawn as a point, each edge is drawn as a horizontal or vertical line segment, and each face is drawn as a rectangle. A planar graph is said to have a rectangular drawing if at least one of its plane embeddings has a rectangular drawing. In this paper we give a linear-time algorithm to examine whether a planar graph $G$ of the maximum degree three has a rectangular drawing or not, and to find a rectangular drawing of $G$ if it exists.
\end{abstract}

\section{Introduction}

The necessity of automatic graph drawing in various areas of science and technology has accelerated the development of many graph drawing styles, and different algorithms have been developed for producing these graph drawing styles [DETT99]. Among different drawing styles a "rectangular drawing" has attracted much attention due to its applications in VLSI floorplanning [KK84, L90, RNN98, RNN02, RNN02, TTSS91] and architectural floorplanning [MKI00. A plane graph is a planar graph with a fixed embedding. A rectangular drawing of a plane graph $G$ is a drawing of $G$ in which each vertex is drawn as a point, each edge is drawn as a horizontal or vertical line segment without edge-crossings, and each face is drawn as a rectangle. (See Figure 1(a).) Not every plane graph has a rectangular drawing. We denote by $\Delta$ the maximum degree of $G$. If a plane graph $G$ has a rectangular drawing, then $\Delta \leq 4$ and $G$ must be biconnected and have four or more vertices of degree 2 on the outer face. Thomassen [T84] obtained a necessary and sufficient condition for a plane graph of $\Delta \leq 3$ to have a rectangular drawing when four vertices of degree two on the outer face are designated as convex corners of a rectangular drawing of the outer face. Linear-time algorithms are given in [BS88, H93, KH97, RNN98] to obtain a rectangular drawing of such

\footnotetext{
* This work is supported by JSPS grants.
} 
a plane graph. The plane graph in Figure 1(b) has a rectangular drawing for the four designated corners $a, f, i$, and $l$ as illustrated in Figure 1(a). Rahman et al. RNN02 gave a necessary and sufficient condition for a plane graph of $\Delta \leq 3$ to have a rectangular drawing when no vertex is designated as a corner, and developed a linear-time algorithm to find a rectangular drawing of a plane graph if it exits.

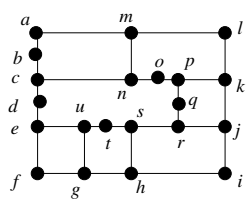

(a)

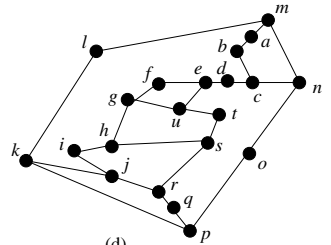

(d)
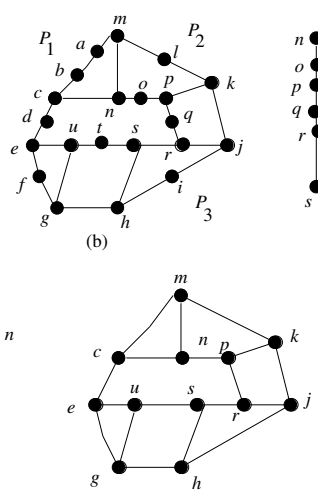

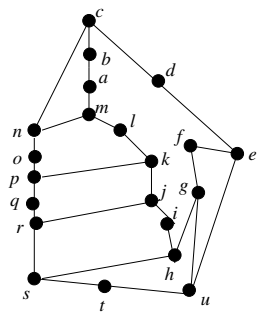

(c)

Fig. 1. A rectangular drawing (a) and three different embeddings (b), (c) and (d) of the same graph which is subdivision of the graph in (e).

A planar graph $G$ is said to have a rectangular drawing if at least one of the plane embeddings of $G$ has a rectangular drawing. Figures 1(b), (c) and (d) depict three different plane embedings of the same planar graph. Among them only the embedding in Figure 1(b) has a rectangular drawing as illustrated in Figure 1(a). Thus the planar graph has a rectangular drawing. A rectangular drawing of a planar graph with $\Delta \leq 3$ has practical application in VLSI floorplanning [KK84, L90, RNN98, RNN02] and architectural floorplanning [MKI00]. Examining whether a planar graph $G$ of $\Delta \leq 3$ has a rectangular drawing is not a trivial problem, since $G$ may have an exponential number of plane embeddings in general. A straightforward algorithm checking each of all the embeddings by the linear algorithm above in [RNN02] does not run in polynomial time. It has thus been desired to obtain an efficient algorithm. In this paper we give a lineartime algorithm to examine whether a planar graph $G$ of $\Delta \leq 3$ has a rectangular drawing and find a rectangular drawing of $G$ if it exists.

We first consider the case where $G$ is a "subdivision" of a planar 3-connected cubic graph, and then consider the case where $G$ is not a "subdivision" of a planar 3-connected cubic graph. The plane graph in Figure 1(b) is a subdivision of the planar 3-connected cubic graph in Figure 1(e). A subdivision $G$ of a planar 3 -connected cubic graph has exactly one embedding for each face embedded as 
the outer face [NC88]. Hence $G$ has an $O(n)$ number of embeddings, one for each chosen outer face. Thus, the straightforward algorithm takes time $O\left(n^{2}\right)$ to examine whether the planar graph $G$ has a rectangular drawing. We however obtain a necessary and sufficient condition for a subdivision $G$ of a planar 3connected cubic graph to have a rectangular drawing, which leads to a linear-time algorithm to examine whether the planar graph $G$ has a rectangular drawing. If $G$ is not a subdivision of a planar 3-connected cubic graph, then $G$ may have an exponential number of embeddings, and hence a straightforward algorithm does not run in polynomial time. We however give a linear-time algorithm to examine whether $G$ has a rectangular drawing or not; we indeed show that it suffices to examine whether only four embeddings of $G$ have rectangular drawings or not.

The rest of the paper is organized as follows. Section 2 describes some definitions and presents preliminary results. Section 3 presents the necessary and sufficient conditions for a planar graph of $\Delta \leq 3$ to have a rectangular drawing. Section 4 presents a linear-time algorithm to find a rectangular drawing of a planar graph $G$ of $\Delta \leq 3$ if it exists. Finally, Section 5 concludes with discussions.

\section{Preliminaries}

In this section we give some definitions and present preliminary results.

Let $G=(V, E)$ be a connected simple graph with vertex set $V$ and edge set $E$. We denote by $d(v)$ the degree of $v$. A graph $G$ is called cubic if $d(v)=3$ for every vertex $v$. For $V^{\prime} \subseteq V, G-V^{\prime}$ denotes a graph obtained from $G$ by deleting all vertices in $V^{\prime}$ together with all edges incident to them. For a subgraph $G^{\prime}$ of $G$, we denote by $G-G^{\prime}$ the graph obtained from $G$ by deleting all vertices in $G^{\prime}$. The connectivity $\kappa(G)$ of a graph $G$ is the minimum number of vertices whose removal results in a disconnected graph or a single-vertex graph $K_{1}$. We say that $G$ is $k$-connected if $\kappa(G) \geq k$. A separation pair of a 2-connected graph $G$ is a pair of vertices whose deletion disconnects $G$. A 3-connected graph has no separation pair.

Let $P=w_{0}, w_{1}, w_{2}, \ldots, w_{k+1}, k \geq 1$, be a path of $G$ such that $d\left(w_{0}\right) \geq$ $3, d\left(w_{1}\right)=d\left(w_{2}\right)=\cdots=d\left(w_{k}\right)=2$, and $d\left(w_{k+1}\right) \geq 3$. Then we call the subpath $P^{\prime}=w_{1}, w_{2}, \ldots, w_{k}$ of $P$ a chain of $G$, and we call vertices $w_{0}$ and $w_{k+1}$ the supports of the chain $P^{\prime}$. If $G$ is a subdivision of a 3-connected graph, then any vertex of degree 2 in $G$ is contained in exactly one of the chains of $G$. Two chains on a cycle are consecutive if they have a common support. Chains $P_{1}=a, b$ and $P_{2}=l$ on the outer face of the plane graph in Figure 1(b) are consecutive, while $P_{1}$ and $P_{3}=i$ are not.

Subdividing an edge $(u, v)$ of a graph $G$ is the operation of deleting the edge $(u, v)$ and adding a path $u\left(=w_{0}\right), w_{1}, w_{2}, \ldots, w_{k}, v\left(=w_{k+1}\right)$ through new vertices $w_{1}, w_{2}, \ldots, w_{k}, k \geq 1$, of degree 2 . A graph $G$ is said to be a subdivision of a graph $G^{\prime}$ if $G$ is obtained from $G^{\prime}$ by subdividing some of the edges of $G^{\prime}$.

A graph $G$ is called cyclically 4-edge-connected if the removal of any three or fewer edges leaves a graph such that exactly one of the connected components has a cycle T92]. 
Let $G$ be a planar biconnected graph, and let $\Gamma$ be a plane embedding of $G$. The contour of a face is a cycle of $G$, and is simply called a face or a facial cycle. We denote by $F_{o}(\Gamma)$ the outer face of $\Gamma$. For a cycle $C$ of $\Gamma$, we call the plane subgraph of $\Gamma$ inside $C$ (including $C$ ) the inner subgraph $\Gamma_{I}(C)$ for $C$, and call the plane subgraph of $\Gamma$ outside $C$ (including $C$ ) the outer subgraph $\Gamma_{O}(C)$ for $C$. An edge of $G$ which is incident to exactly one vertex of a cycle $C$ and located outside $C$ is called a leg of $C$. The vertex of $C$ to which a leg is incident is called a leg-vertex of $C$. A cycle $C$ in $\Gamma$ is called a $k$-legged cycle of $\Gamma$ if $C$ has exactly $k$ legs in $\Gamma$ and there is no edge which joins two vertices on $C$ and is located outside $C$. The set of $k$ legs of a $k$-legged cycle in $\Gamma$ corresponds to a "cutset" of $k$ edges for $k=2$ or 3. We call a face $F$ of $\Gamma$ a peripheral face for a 3-legged cycle $C$ in $\Gamma$ if $F$ is in $\Gamma_{O}(C)$ and the contour of $F$ contains some edges on $C$. Clearly there are exactly three peripheral faces for any 3-legged cycle in $\Gamma$. A $k$-legged cycle $C$ is called a minimal k-legged cycle if $G_{I}(C)$ does not contain any other $k$-legged cycle of $G$. We say that cycles $C$ and $C^{\prime}$ in $\Gamma$ are independent if $\Gamma_{I}(C)$ and $\Gamma_{I}\left(C^{\prime}\right)$ have no common vertex. A set $\mathcal{S}$ of cycles is independent if any pair of cycles in $\mathcal{S}$ are independent. A plane biconnected graph $G$ may have a separation pair, and the pair of leg-vertices of any 2-legged cycle in $G$ is a separation pair. A cycle $C$ in a plane embedding $\Gamma$ of $G$ is called regular if the plane graph $\Gamma-\Gamma_{I}(C)$ has a cycle. Clearly a 2-legged cycle $C$ in $\Gamma$ is not regular if and only if $\Gamma-\Gamma_{I}(C)$ is a chain of $G$, while a 3-legged cycle $C$ is not regular if and only if $\Gamma-\Gamma_{I}(C)$ contains exactly one vertex that has degree 3 in $G$. Let $G$ be a subdivision of a planar 3-connected cubic graph, then $\Gamma$ has no regular 2-legged cycle, but may have a regular 3-legged cycle, and $\Gamma$ has no regular 3-legged cycle if and only if $G$ is cyclically 4-edge-connected.

Ungar U53] showed that any plane embedding $\Gamma$ of a cyclically 4-edgeconnected planar cubic graph $G$ has a rectangular drawing if four vertices of degree 2 are inserted on some edges on the outer face $F_{o}(\Gamma)$. Generalizing the results of Ungar, Thomassen T84 obtained a necessary and sufficient condition for a plane embedding of a planar graph $G$ with $\Delta \leq 3$ to have a rectangular drawing when four vertices of degree 2 on $F_{o}(\Gamma)$ are designated as corners for a rectangular drawing. Generalizing the result of Thomassen, Rahman et al. RNN02 gave a necessary and sufficient condition for a plane embedding of a planar graph $G$ with $\Delta \leq 3$ to have a rectangular drawing when no vertex is designated as a corner, and developed a linear-time algorithm to find a rectangular drawing of $G$ if it exits, as in the following Lemma 1 .

Lemma 1. RNN02] A plane embedding $\Gamma$ of a planar biconnected graph $G$ of $\Delta \leq 3$ has a rectangular drawing if and only if $\Gamma$ satisfies the following four conditions:

(a) there are four or more vertices of degree 2 on $F_{o}(\Gamma)$;

(b) every 2-legged cycle in $\Gamma$ contains at least two vertices of degree 2 on $F_{o}(\Gamma)$;

(c) every 3-legged cycle in $\Gamma$ contains at least one vertex of degree 2 on $F_{o}(\Gamma)$; and

(d) if an independent set $\mathcal{S}$ of cycles in $\Gamma$ consists of $c_{2}$ 2-legged cycles and $c_{3}$ 3-legged cycles, then $2 c_{2}+c_{3} \leq 4$. 
Furthermore one can examine in linear time whether $\Gamma$ satisfies the condition above, and if $\Gamma$ does then one can find a rectangular drawing of $\Gamma$ in linear time.

Although the results above for plane embeddings are known, it is difficult to examine whether a planar graph has a rectangular drawing or not, since a planar graph may have an exponential number of plane embeddings in general. The following fact is known for subdivisions of planar 3-connected cubic graphs.

Fact 2 NC88 Let $G$ be a subdivision of a 3-connected planar graph. Then there is exactly one embedding of $G$ for each face embedded as the outer face. Furthermore, for any two plane embeddings $\Gamma$ and $\Gamma^{\prime}$ of $G$, any facial cycle in $\Gamma$ is a facial cycle in $\Gamma^{\prime}$.

\section{Rectangular Drawings of Planar Graphs}

In this section we give necessary and sufficient conditions for a planar graph of $\Delta \leq 3$ to have a rectangular drawing. In Section 3.1 we consider the case where $G$ is a subdivision of a planar 3-connected cubic graph, and in Section 3.2 the other case.

\subsection{Case for a Subdivision of a Planar 3-Connected Cubic Graph}

Let $G$ be a subdivision of a planar 3-connected cubic graph. Then by Fact $2 G$ has an $O(n)$ number of embeddings, one for each chosen outer face. Examining by the linear algorithm in Lemma 1 whether the four conditions hold for each of the $O(n)$ embeddings, one can examine in time $O\left(n^{2}\right)$ whether $G$ has a rectangular drawing. However, we obtain the following necessary and sufficient condition for $G$ to have a rectangular drawing, which leads to a linear-time algorithm.

Theorem 1. Let $G$ be a subdivision of a planar 3-connected cubic graph, and let $\Gamma$ be an arbitrary plane embedding of $G$.

(a) Suppose first that $G$ is cyclically 4-edge-connected, that is, $\Gamma$ has no regular 3 -legged cycle. Then the planar graph $G$ has a rectangular drawing if and only if $\Gamma$ has a face $F$ such that

(i) F contains at least four vertices of degree 2;

(ii) there are at least two chains on F; and

(iii) if there are exactly two chains on $F$, then they are not consecutive and each of them contains at least two vertices.

(b) Suppose next that $G$ is not cyclically 4-edge connected, that is, $\Gamma$ has a regular 3-legged cycle $C$. Let $F_{1}, F_{2}$ and $F_{3}$ be the three peripheral faces for $C$, and let $\Gamma_{1}, \Gamma_{2}$ and $\Gamma_{3}$ be the plane embeddings of $G$ taking $F_{1}, F_{2}$ and $F_{3}$, respectively, as the outer face. Then the planar graph $G$ has a rectangular drawing if and only if at least one of the three embeddings $\Gamma_{1}, \Gamma_{2}$ and $\Gamma_{3}$ has a rectangular drawing. 
Before giving a proof of Theorem 1. we observe the following lemma on subdivisions of planar 3-connected cubic graphs.

Lemma 3. Let $G$ be a subdivision of a planar 3-connected cubic graph $G^{\prime}$, and let $\Gamma$ be an arbitrary plane embedding of $G$. Then the following (a) - (c) hold.

(a) If $C$ is a 2-legged cycle in $\Gamma$, then the legs $e_{1}$ and $e_{2}$ and the leg-vertices $v_{1}$ and $v_{2}$ of $C$ are on the outer face $F_{o}(\Gamma)$, and the set of all vertices not in $\Gamma_{I}(C)$ induces a chain of $G$ on $F_{o}(\Gamma)$ with supports $v_{1}$ and $v_{2}$.

(b) For any chain $P$ on $F_{o}(\Gamma)$, the outer face of the plane graph $\Gamma-P$ is a 2-legged cycle in $\Gamma$.

(c) Any pair of 2-legged cycles in $\Gamma$ are not independent.

We are now ready to give a proof of Theorem 1 .

Proof of Theorem 1(a): Necessity: Assume that $G$ is cyclically 4-edge-connected and has a rectangular drawing. Then a plane embedding $\Gamma^{\prime}$ of $G$ has a rectangular drawing.

The four corner vertices of a rectangular drawing of $F_{o}\left(\Gamma^{\prime}\right)$ have degree 2, and hence $F_{o}\left(\Gamma^{\prime}\right)$ has at least four vertices of degree 2 .

If all the four corner vertices are contained in the same chain on $F_{o}\left(\Gamma^{\prime}\right)$, then $G$ would not have a rectangular drawing. Therefore, there are two or more chains on $F_{o}\left(\Gamma^{\prime}\right)$.

Suppose that there are exactly two chains on $F_{o}\left(\Gamma^{\prime}\right)$. Then clearly each of them contains exactly two of the four corner vertices of degree 2 ; otherwise, $\Gamma^{\prime}$ would not have a rectangular drawing. Furthermore, the two chains must be non-consecutive; otherwise, $\Gamma^{\prime}$ would have a 3-legged cycle $C$ containing no vertex of degree 2 on $F_{o}\left(\Gamma^{\prime}\right)$, contrary to Lemma T(c).

Thus the outer face $F_{o}\left(\Gamma^{\prime}\right)$ satisfies Conditions (i) - (iii) of Theorem 11(a). By Fact 2 the outer face $F_{o}\left(\Gamma^{\prime}\right)$ corresponds to a face $F$ of the arbitrary plane embedding $\Gamma$ of $G$. Therefore, the face $F$ in $\Gamma$ satisfies Conditions (i), (ii) and (iii) of Theorem 1 .

Sufficiency: We give a constructive proof for the sufficiency of Theorem 1(a). Assume that $\Gamma$ has a face $F$ satisfying Conditions (i) - (iii) of Theorem 11(a). Let $\Gamma^{\prime}$ be an embedding of $G$ such that $F=F_{o}\left(\Gamma^{\prime}\right)$. It is sufficient to prove that $\Gamma^{\prime}$ satisfies Conditions (a) - (d) in Lemma 1.

(a) By Condition (i) in Theorem 1(a) $F_{o}\left(\Gamma^{\prime}\right)=F$ contains at least four vertices of degree 2, and hence $\Gamma^{\prime}$ satisfies Condition (a) in Lemma 1 .

(b) We now show that $\Gamma^{\prime}$ satisfies Condititon (b) in Lemma 1 that is, every 2-legged cycle $C$ in $\Gamma^{\prime}$ contains at least two vertices of degree 2 on $F_{o}\left(\Gamma^{\prime}\right)$. By Condition (ii) in Theorem 1(a) there are at least two chains on $F_{o}\left(\Gamma^{\prime}\right)$, and hence we have the following two cases to consider.

Case 1: there are exactly two chains $P_{1}$ and $P_{2}$ on $F_{o}\left(\Gamma^{\prime}\right)$.

By Lemmas 3(a) - (c) $\Gamma^{\prime}$ has exactly two 2-legged cycles $C_{1}$ and $C_{2}$. Clearly each of the 2-legged cycles $C_{1}$ and $C_{2}$ contains exactly one chain. By Condition (iii) of Theorem 1(a) each of the two chains $P_{1}$ and $P_{2}$ contains at least two vertices of degree 2 . Therefore, each 2-legged cycle contains at least two vertices of degree 2 on $F_{o}\left(\Gamma^{\prime}\right)$. Thus $\Gamma^{\prime}$ satisfies Condition (b) of Lemma 1 . 
Case 2: there are more than two chains on $F_{o}\left(\Gamma^{\prime}\right)$.

Assume that there are exactly $r$ chains on $F_{o}\left(\Gamma^{\prime}\right)$ and $r \geq 3$. Then each 2-legged cycle contains $r-1(\geq 2)$ chains on $F_{o}\left(\Gamma^{\prime}\right)$, and hence contains at least two vertices of degree 2 on $F_{o}\left(\Gamma^{\prime}\right)$. Thus $\Gamma^{\prime}$ satisfies Condition (b) of Lemma 1.

(c) We next show that $\Gamma^{\prime}$ satisfies Condition (c) in Lemma 1 that is, every 3-legged cycle $C$ in $\Gamma^{\prime}$ contains at least one vertex of degree 2 on $F_{o}\left(\Gamma^{\prime}\right)$. Since $G$ is cyclically 4-edge connected, $C$ is not regular and hence $\Gamma^{\prime}-\Gamma_{I}^{\prime}(C)$ has no cycle. Therefore, $C$ contains edges on $F_{o}\left(\Gamma^{\prime}\right)$, two of the three leg-vertices of $C$, say $u$ and $v$, are on $F_{o}\left(\Gamma^{\prime}\right)$, and $\Gamma^{\prime}-\Gamma_{I}^{\prime}(C)$ contains exactly one vertex $w$ that has degree three in $G$. Clearly $w$ is on $F_{o}\left(\Gamma^{\prime}\right)$. The three vertices $u, w$ and $v$ divide $F_{o}\left(\Gamma^{\prime}\right)$ to three paths $P_{1}, P_{2}$ and $P_{3} ; P_{1}$ goes from $u$ to $w$ on $F_{o}\left(\Gamma^{\prime}\right), P_{2}$ from $w$ to $v$, and $P_{3}$ from $v$ to $u$. By Condition (ii) in Theorem 1(a) there are at least two chains on $F=F_{o}\left(\Gamma^{\prime}\right)$. We now have the following two cases.

Case 1: at most one of $P_{1}$ and $P_{2}$ contains a chain.

In this case there are at least one chain on $P_{3}$ since there are at least two chains on $F_{o}\left(\Gamma^{\prime}\right)$. Therefore, $C$ contains at least one vertex of degree 2 on $F_{o}\left(\Gamma^{\prime}\right)$. Thus $\Gamma^{\prime}$ satisfies Condition (c) in Lemma 1. Case 2: each of $P_{1}$ and $P_{2}$ contains a chain.

In this case the two chains on $P_{1}$ and $P_{2}$ are consecutive. Therefore by Condition (iii) in Theorem 1(a) $F_{o}\left(\Gamma^{\prime}\right)$ contains three or more chains, and hence there is at least one chain on $P_{3}$. Thus $C$ contains at least one vertex of degree 2 on $F_{o}\left(\Gamma^{\prime}\right)$, and hence $\Gamma^{\prime}$ satisfies Condition (c) in Lemma 1,

(d) We finally show that $\Gamma^{\prime}$ satisfies Condition (d) in Lemma 1. Let $\mathcal{S}$ be any independent set of cycles in $\Gamma^{\prime}$. Then $c_{2} \leq 1$ by Lemma 3(c).

We now claim that $c_{3} \leq 1$. Otherwise, there are two different 3-legged cycles $C$ and $C^{\prime}$ in $\mathcal{S}$. By (c) above each of $C$ and $C^{\prime}$ contains at least one vertex of $F_{o}\left(\Gamma^{\prime}\right)$. Since $G$ is cyclically 4-edge connected, $C$ and $C^{\prime}$ are not regular and hence each of the plane graphs $\Gamma^{\prime}-\Gamma_{I}^{\prime}(C)$ and $\Gamma^{\prime}-\Gamma_{I}^{\prime}\left(C^{\prime}\right)$ contains exactly one vertex that has degree 3 in $G$. Then clearly $\Gamma_{I}^{\prime}(C)$ and $\Gamma_{I}^{\prime}\left(C^{\prime}\right)$ have common vertices, contrary to the assumption that $\mathcal{S}$ is an independent set of cycles.

Since $c_{2} \leq 1$ and $c_{3} \leq 1$, we have $2 c_{2}+c_{3} \leq 3$. Thus $\Gamma^{\prime}$ satisfies Condition (d) of Lemma 1 .

Q.E.D.

Proof of Theorem 1(b): Since the proof for the sufficiency is obvious, we give a proof for the necessity. Suppose that $\Gamma$ has a regular 3-legged cycle $C$ and that the planar graph $G$ has a rectangular drawing. Then there is a plane embedding $\Gamma^{\prime}$ of $G$ which has a rectangular drawing. Let $F$ be the face of $\Gamma$ corresponding to $F_{o}\left(\Gamma^{\prime}\right)$. It suffices to show that $F$ is one of the three peripheral faces $F_{1}, F_{2}$ and $F_{3}$ for $C$ in $\Gamma$.

We first consider the case where $C$ contains an edge on $F_{o}(\Gamma)$. Let $C^{\prime}$ be the cycle in $\Gamma-\Gamma_{I}(C)$ such that $\Gamma_{I}\left(C^{\prime}\right)$ has the maximum number of edges. One can observe that $C^{\prime}$ is a 3-legged cycle in $\Gamma$, and any face of $\Gamma$ other than $F_{1}$, $F_{2}$ and $F_{3}$ is in $\Gamma_{I}(C)$ or $\Gamma_{I}\left(C^{\prime}\right)$. Therefore it is sufficient to prove that $F$ is neither in $\Gamma_{I}(C)$ nor in $\Gamma_{I}\left(C^{\prime}\right)$. If $F$ is in $\Gamma_{I}(C)$, then a 3-legged cycle $C^{\prime}$ in $\Gamma^{\prime}$ contains no vertex on $F_{o}\left(\Gamma^{\prime}\right)=F$, a contradiction to Lemma 1(c). Similarly, if 
$F$ is in $\Gamma_{I}\left(C^{\prime}\right)$, then a 3-legged cycle $C$ in $\Gamma^{\prime}$ contains no vertex on $F_{o}\left(\Gamma^{\prime}\right)=F$, a contradiction to Lemma 1(c).

We next consider the case where $C$ does not contain any edge on $F_{o}(\Gamma)$. Let $C^{\prime}$ be the cycle in $\Gamma-\Gamma_{I}(C)$ such that $\Gamma_{I}\left(C^{\prime}\right)$ includes $\Gamma_{I}(C)$ and has the minimum number of edges. Any face of $\Gamma$ other than $F_{1}, F_{2}$ and $F_{3}$ is in $\Gamma_{I}(C)$ or $\Gamma_{O}\left(C^{\prime}\right)$. Therefore it is sufficient to prove that $F$ is neither in $\Gamma_{I}(C)$ nor in $\Gamma_{O}\left(C^{\prime}\right)$. If $F$ is in $\Gamma_{I}(C)$, then a 3-legged cycle $C^{\prime}$ in $\Gamma^{\prime}$ contains no vertex on $F_{o}\left(\Gamma^{\prime}\right)=F$, a contradiction to Lemma 1)(c). If $F$ is in $\Gamma_{O}\left(C^{\prime}\right)$, then a 3-legged cycle $C$ in $\Gamma^{\prime}$ contains no vertex on $F_{o}\left(\Gamma^{\prime}\right)=F$, a contradiction to Lemma 1 $(\mathrm{c})$. $\mathcal{Q} . \mathcal{E} . \mathcal{D}$.

Theorem 1 immediately yields the following algorithm to examine whether a subdivision $G$ of a planar 3-connected cubic graph has a rectangular drawing and to find a rectangular drawing of $G$ if it exists.

\section{Algorithm Subdivision-Draw $(G)$ \\ begin}

Let $\Gamma$ be any plane emebedding of $G$;

1 Examine whether $\Gamma$ has a regular 3-legged cycle $C$;

2 if $\Gamma$ has no regular 3 -legged cycle then

begin $\{G$ is cyclically 4 -edge-connected. $\}$

Find a face $F$ in $\Gamma$ which satisfies Conditions (i)-(iii)

in Theorem 1)(a);

4

if $\Gamma$ has such a face $F$ then

\section{begin}

5

6

Let $\Gamma^{\prime}$ be the plane embedding of $G$ whose outer face is $F$;

7

8

end

9 else $\{\Gamma$ has a regular 3-legged cycle, and $G$ is not cyclically 4-edge-connected. $\}$

\section{begin}

Find a rectangular drawing of $\Gamma^{\prime}$ by the algorithm in [RNN02] end

else

let $C$ be a regular 3-legged cycle;

Let $F_{1}, F_{2}$ and $F_{3}$ be the three peripheral faces of $C$;

Let $\Gamma_{1}, \Gamma_{2}$ and $\Gamma_{3}$ be three plane embeddings of $G$ taking

$F_{1}, F_{2}$, and $F_{3}$ as the outer face, respectively;

Examine whether $\Gamma_{1}, \Gamma_{2}$ and $\Gamma_{3}$ have rectangular drawings by

the algorithm in [RNN02]; else

Find a rectangular drawing of $\Gamma_{1}$ by the algorithm in [RNN02]

$$
\text { end } G \text { has no rectangular drawing; }
$$

end. 
Theorem 2. Algorithm Subdivision-Draw examines in linear time whether a subdivision $G$ of a planar 3-connected cubic graph has a rectangular drawing, and finds a rectangular drawing of $G$ in linear time if it exists.

\subsection{The Other Case}

In this section we assume that $G$ is a planar biconnected graph of $\Delta \leq 3$ but is not a subdivision of a 3 -connected cubic graph, and give a linear-time algorithm to examine whether $G$ has a rectangular drawing and to find a rectangular drawing of $G$ if it exists.

Let $\Gamma$ be an arbitrary plane embedding of $G$. It is trivial to examine whether $G$ has a rectangular drawing if $\Gamma$ has at most two inner faces. We may thus assume that $\Gamma$ has three or more inner faces. Since $G$ is not a subdivision of a planar 3-connected cubic graph, $\Gamma$ has a regular 2-legged cycle.

Let $C_{1}, C_{2}, \cdots, C_{l}$ be the regular 2-legged cycles in $\Gamma$, and let $x_{i}, y_{i}, 1 \leq i \leq l$, be the two leg-vertices of $C_{i}$. One can easily observe that $l=O(n)$ if $G$ has $n$ vertices [HT73]. If the planar graph $G$ has a rectangular drawing, then a plane embedding $\Gamma^{\prime}$ of $G$ has a rectangular drawing. The outer face $F_{o}\left(\Gamma^{\prime}\right)$ must contain all vertices $x_{1}, y_{1}, x_{2}, y_{2}, \cdots, x_{l}, y_{l}$; otherwise, $\Gamma^{\prime}$ would not have a rectangular drawing as known from Lemma 1(b). Construct a graph $G^{+}$from $G$ by adding a dummy vertex $z$ and dummy edges $\left(x_{i}, z\right)$ and $\left(y_{i}, z\right)$ for all indices $i, 1 \leq i \leq l$. If $G^{+}$is not planar, then $G$ has no rectangular drawing. We thus assume that $G^{+}$is planar and has an embedding $\Gamma^{+}$such that $z$ is embedded on the outer face.

We delete from $\Gamma^{+}$the dummy vertex $z$ and all dummy edges incident to $z$, and let $\Gamma^{*}$ be the resulting plane embedding of $G$, in which $F_{o}\left(\Gamma^{*}\right)$ contains all vertices $x_{1}, y_{1}, x_{2}, y_{2}, \cdots, x_{l}, y_{l}$. If $\Gamma^{*}$ has three or more independent 2-legged cycles, then any plane embedding $\Gamma^{\prime \prime}$ of $G$ whose outer face contains all vertices $x_{1}, y_{1}, x_{2}, y_{2}, \cdots, x_{l}, y_{l}$ has three or more independent 2-legged cycles, and hence by Lemma 1(d) $\Gamma^{\prime \prime}$ has no rectangular drawing, and consequently the planar graph $G$ has no rectangular drawing. We may thus assume that $\Gamma^{*}$ has two or less independent 2-legged cycle.

Since $\Gamma$ has a regular 2-legged cycle, $\Gamma^{*}$ has two or more independent 2legged cycles. Thus $\Gamma^{*}$ has exactly two independent 2-legged cycles $C_{1}$ and $C_{2}$. We may assume without loss of generality that $C_{1}$ and $C_{2}$ are minimal 2-legged cycles, as illustrated in Figure 2(a). If we flip $\Gamma_{I}^{*}(C)$ for any 2-legged cycle $C$ other than $C_{1}$ and $C_{2}$, then the outer face of the resulting embedding does not contain the leg-vertices of $C_{1}$ or $C_{2}$. By flipping $\Gamma_{I}^{*}\left(C_{1}\right)$ or $\Gamma_{I}^{*}\left(C_{2}\right)$ around the leg-vertices of $C_{1}$ or $C_{2}$, we have four different embeddings $\Gamma_{1}\left(=\Gamma^{*}\right), \Gamma_{2}, \Gamma_{3}$ and $\Gamma_{4}$ such that each $F_{o}\left(\Gamma_{i}\right), 1 \leq i \leq 4$, contains all vertices $x_{1}, y_{1}, x_{2}, y_{2}, \cdots, x_{l}, y_{l}$. $\Gamma_{1}$ and $\Gamma_{2}$ are illustrated in Figure 2. Clearly, only these four embeddings $\Gamma_{1}, \Gamma_{2}$, $\Gamma_{3}$ and $\Gamma_{4}$ have all vertices $x_{1}, y_{1}, x_{2}, y_{2}, \cdots, x_{l}, y_{l}$ on the outer face. Thus $G$ has a rectangular drawing if and only if any of $\Gamma_{1}, \Gamma_{2}, \Gamma_{3}$ and $\Gamma_{4}$ has a rectangular drawing. We thus have the following theorem. 

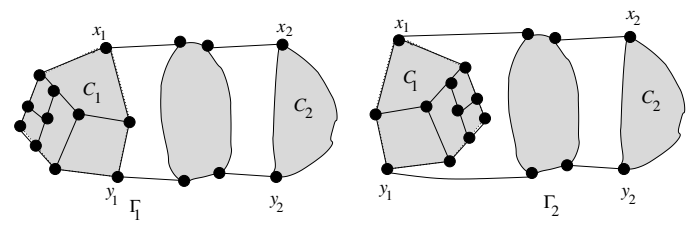

Fig. 2. (a) $\Gamma_{1}=\Gamma^{*}$, and (b) $\Gamma_{2}$ is obtained by flipping $\Gamma_{I}^{*}\left(C_{1}\right)$ only.

Theorem 3. Let $G$ be a planar biconnected graph of $\Delta \leq 3$ which is not a subdivision of a planar 3-connected cubic graph. Let $\Gamma$ be a planar embedding of $G$ such that every 2-legged cycle in $\Gamma$ has leg-vertices on $F_{o}(\Gamma)$, and let $C_{1}$ and $C_{2}$ be the two minimal 2-legged cycles in $\Gamma$. Let $\Gamma_{1}(=\Gamma), \Gamma_{2}, \Gamma_{3}$, and $\Gamma_{4}$ be the four embeddings of $G$ obtained from $\Gamma$ by flipping $\Gamma_{I}\left(C_{1}\right)$ or $\Gamma_{I}\left(C_{2}\right)$ around the leg-vertices of $C_{1}$ and $C_{2}$. Then $G$ has a rectangular drawing if and only if at least one of the four embeddings $\Gamma_{1}, \Gamma_{2}, \Gamma_{3}$, and $\Gamma_{4}$ has a rectangular drawing.

\section{Algorithm}

In this section we formally describe our algorithm Planar-Rectangular-Draw to examine whether a planar graph $G$ of $\Delta \leq 3$ has a rectangular drawing and to find a rectangular drawing of $G$ if it exists.

\section{Algorithm Planar-Rectangular-Draw $(G)$ \\ begin}

Let $\Gamma$ be any plane emebedding of $G$;

1 Examine whether $\Gamma$ has a regular 2-legged cycle;

2 if $\Gamma$ has no regular 2-legged cycle then

$\{G$ is a subdivision of a planar 3-connected cubic graph. $\}$

Examine by Algorithm Subdivision-Draw whether $G$ has a rectangular drawing and find a rectangular drawing of $G$ if it exists;

else $\{G$ is not a subdivision of a planar 3 -connected cubic graph. $\}$

\section{begin}

let $x_{1}, y_{1}, x_{2}, y_{2}, \cdots, x_{l}, y_{l}$ be the leg-vertices of regular 2-legged cycles $C_{1}, C_{2}, \cdots, C_{l}$ in $\Gamma$; dummy edges $\left(x_{i}, z\right)$ and $\left(y_{i}, z\right)$ for all indices $i, 1 \leq i \leq l$; Examine whether $G^{+}$is planar;

if $G^{+}$is not planar then

$G$ has no rectangular drawing else

\section{begin}

Find a planar embedding $\Gamma^{+}$of $G^{+}$such that $z$ is embedded on the outer face;

Delete from $\Gamma^{+}$the dummy vertex $z$ and all dummy edges incident to $z$, 
12

17

and let $\Gamma^{*}$ be the resulting plane graph;

Let $C_{1}$ and $C_{2}$ be the two minimal regular 2-legged cycles in $\Gamma^{*}$;

Let $\Gamma_{1}, \Gamma_{2} \Gamma_{3}$ and $\Gamma_{4}$ be the four plane embeddings of $G$ obtained from $\Gamma^{*}$ by flipping $\Gamma_{I}^{*}\left(C_{1}\right)$ or $\Gamma_{I}^{*}\left(C_{2}\right)$ around the leg-vertices of $C_{1}$ or $C_{2}$;

Examine whether $\Gamma_{1}, \Gamma_{2} \Gamma_{3}$ and $\Gamma_{4}$ have rectangular drawings by the algorithm in [RNN02]; if $\Gamma_{1}, \Gamma_{2}, \Gamma_{3}$ or $\Gamma_{4}$, say $\Gamma_{1}$, has a rectangular drawing then else Find a rectangular drawing of $\Gamma_{1}$ by the algorithm in [RNN02]

$G$ has no rectangular drawing;

end.

$$
\text { end }
$$

We now have the following theorem on Algorithm Planar-RectangularDraw.

Theorem 4. Let $G$ be a planar biconnected graph of $\Delta \leq 3$. Then Algorithm Planar-Rectangular-Draw examines in linear time whether $G$ has a rectangular drawing and finds a rectangular drawing of $G$ if it exists.

\section{Conclusions}

In this paper we give a linear-time algorithm to examine whether a planar graph $G$ of $\Delta \leq 3$ has a rectangular drawing and to find a rectangular drawing of $G$ if it exists.

A box-rectangular drawing of a plane graph $G$ is a drawing of $G$ such that each vertex is drawn as a (possibly degenerated) rectangle, called a box, each edge is drawn as a horizontal or a vertical line segment and the contour of each face is drawn as a rectangle. Linear-time algorithms are known to determine whether a plane graph has a box-rectangular drawing and find a box-rectangular drawing if it exists [RNN00, H01]. A planar graph is said to have a box-rectangular drawing if one of its plane embeddings has a box-rectangular drawing. A straightforward algorithm checking each of all embeddings by a linear algorithm in [RNN00, H01] does not run in polynomial time. Extending the algorithm in this paper, one can obtain a linear algorithm to examine whether a planar graph has a box-rectangular drawing and find a box-rectangular drawing if it exists.

\section{References}

BS88. J. Bhasker and S. Sahni, A linear algorithm to find a rectangular dual of a planar triangulated graph, Algorithmica, 3, pp. 247-278, 1988.

DETT99. G. Di Battista, P. Eades, R. Tamassia and I. G. Tollis. Graph Drawing, Prentice Hall, Upper Saddle River, NJ, 1999. 
H93. X. He, On finding the rectangular duals of planar triangulated graphs, SIAM J. Comput., 22(6), pp. 1218-1226, 1993.

H01. X. He, A simple linear time algorithm for proper box rectangular drawings of plane graphs, Journal of Algorithms, 40(1), pp. 82-101, 2001.

HT73. J. E. Hopcroft and R. E. Tarjan, Dividing a graph into triconnected components, SIAM J. Comput., 2(3), pp. 135-158, 1973.

KH97. G. Kant and X. He, Regular edge labeling of 4-connected plane graphs and its applications in graph drawing problems, Theoretical Computer Science, 172, pp. 175-193, 1997.

KK84. K. Kozminski and E. Kinnen, An algorithm for finding a rectangular dual of a planar graph for use in area planning for VLSI integrated circuits, Proc. 21st DAC, Albuquerque, pp. 655-656, 1984.

L90. T. Lengauer, Combinatirial Algorithms for Integrated Circuit Layout, John Wiley \& Sons, Chichester, 1990.

MKI00. S. Munemoto, N. Katoh and G. Imamura, Finding an optimal floor layout based on an orthogonal graph drawing algorithm, J. Archit. Plann. Enviroment Eng. AIJ, No. 524, pp. 279-286, 2000.

NC88. T. Nishizeki and N. Chiba, Planar Graphs: Theory and Algorithms, NorthHolland, Amsterdam, 1988.

RNN98. M. S. Rahman, S. Nakano and T. Nishizeki, Rectangular grid drawings of plane graphs, Comp. Geom. Theo. Appl., 10(3), pp. 203-220, 1998.

RNN99. M. S. Rahman, S. Nakano and T. Nishizeki, A linear algorithm for bendoptimal orthogonal drawings of triconnected cubic plane graphs, Journal of Graph Alg. Appl., 3, No.4, pp. 31-62, 1999.

RNN00. M. S. Rahman, S. Nakano and T. Nishizeki, Box-rectangular drawings of plane graphs, Journal of Algorithms, 37(2), pp. 363-398, 2000.

RNN02. M. S. Rahman, S. Nakano and T. Nishizeki, Rectangular drawings of plane graphs without designated corners, Comp. Geom. Theo. Appl., 21(3), pp. 121-138, 2002.

TTSS91. K. Tani, S. Tsukiyama, S. Shinoda and I. Shirakawa, On area-efficient drawings of rectangular duals for VLSI floor-plan, Mathematical Programming, 52, pp. 29-43, 1991.

T84. C. Thomassen, Plane representations of graphs, (Eds.) J. A. Bondy and U. S. R. Murty, Progress in Graph Theory, Academic Press Canada, pp. 43-69, 1984.

T92. C. Thomassen, Plane cubic graphs with prescribed face areas, Combinatorics, Probability and Computing, 1, pp. 371-381, 1992.

U53. P. Ungar, On diagrams representing maps, J. London Math. Soc., 28, pp. 336-342, 1953. 